
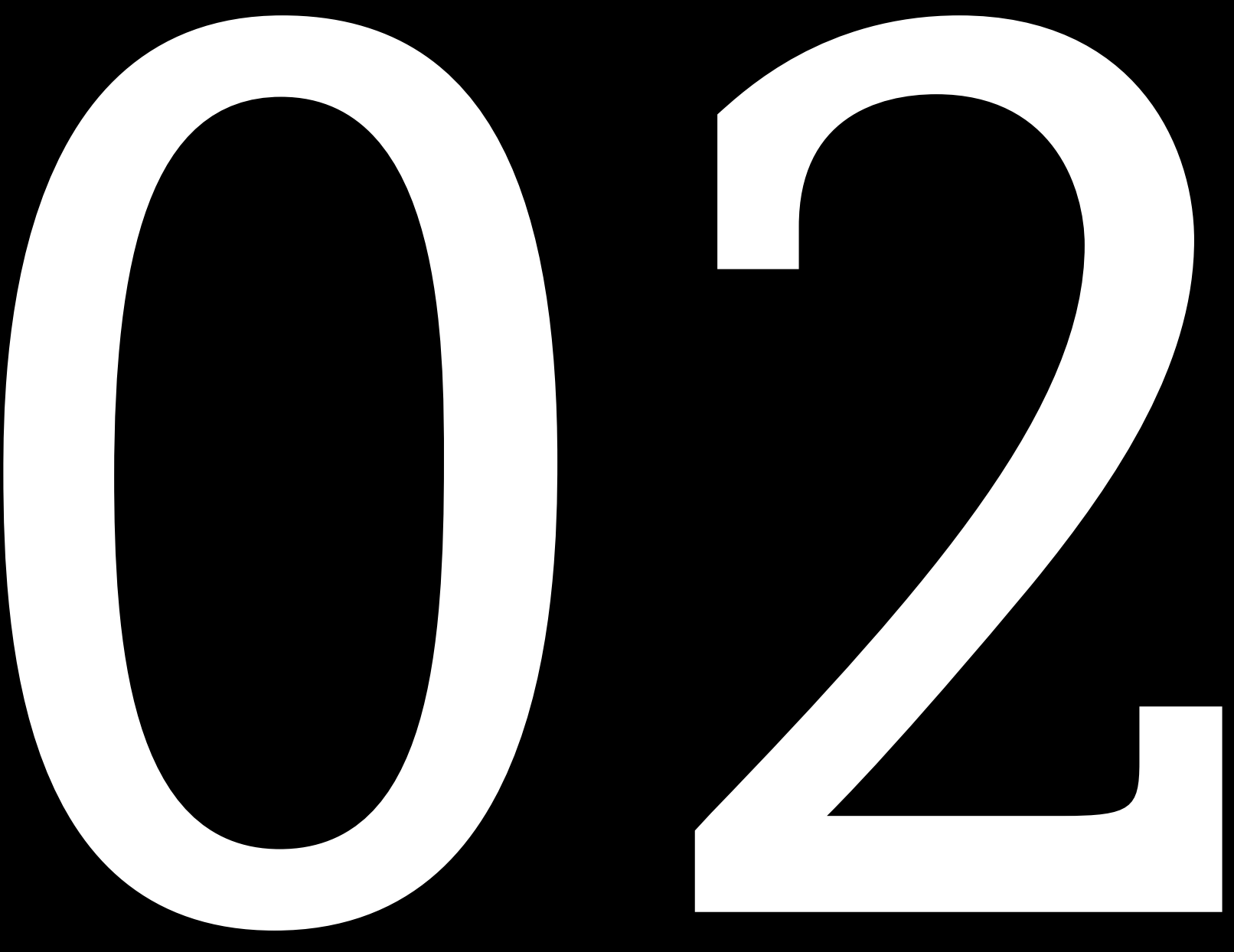

DOI: https://doi.org/10.14483/2422278X.13964 


\section{Pensar la enseñanza de la historia reciente desde la conciencia histórica ${ }^{1}$}

\section{Thoughts on teaching recent history with historical awareness}

\section{Pensando no ensino da história recente a partir da consciência histórica}

\section{Liliana del Pilar Escobar Rincón ${ }^{2}$}

Para citar este artículo: Escobar, L. P. (2019). Pensar la enseñanza de la historia reciente desde la conciencia histórica. Ciudad Paz-ando, 12(2), 21-31. doi: https://doi.org/10.14483/2422278X.13964

Fecha de recepción: 19 de octubre de 2018

Fecha de aprobación: 4 de octubre de 2019

\footnotetext{
1 Este artículo surge como parte de los resultados finales que arroja la tesis doctoral titulada La conciencia histórica y la enseñanza del conflicto armado colombiano: un análisis de los libros de texto escolar de ciencias sociales (1984-2016). Este trabajo doctoral hace parte de la línea de Formación Política y Memoria Social dirigida por el doctor Diego Hernán Arias, el cual tiene como objetivo analizar la formación de la conciencia histórica en la enseñanza de los temas del conflicto armado colombiano desde los libros de texto escolar de ciencias sociales de grado noveno, publicados entre 1984 y 2016. La investigación se orientó desde un enfoque sociocrítico que busca analizar las formas en que se asume, disputa y ejerce el poder en la enseñanza del conflicto armado, utilizando como técnica el análisis documental.

2 Licenciada en Educación Básica con Énfasis en Ciencias Sociales, Universidad Distrital Francisco José de Caldas; Magíster en Investigación Social Interdisciplinaria, Universidad Distrital Francisco José de Caldas. Candidata a Doctora en Educación del Doctorado Institucional en Educación. Correo electrónico: ldescobarr@udistrital.edu.co, ORCID: https://orcid.org/0000-0002-9495-2613
} 


\section{RESUMEN}

Este trabajo, producto de la tesis doctoral titulada La conciencia histórica y la enseñanza del conflicto armado colombiano: un análisis de los libros de texto escolar de ciencias sociales (1984-2016), busca exponer elementos teóricos que permiten comprender la importancia de vincular la categoría de conciencia histórica a apuestas epistemológicas, éticas y políticas que afronta la enseñanza de la historia reciente en la escuela. Para desarrollar la discusión, primero se analizan los retos que implica enseñar los pasados violentos; en un segundo frente, se expone la definición de conciencia histórica, teniendo en cuenta los elementos que la componen; en un tercer punto, se discute el potencial de establecer una relación entre la categoría de conciencia histórica y la enseñanza de la historia reciente. El último apartado recoge los principales puntos de lo expuesto, haciendo hincapié en la importancia de tejer puentes teóricos para comprender diversas narrativas históricas en el escenario escolar. Palabras clave: educación, enseñanza de la historia, formación política, escuela, problema social.

This work, which is the result of the doctoral thesis entitled Historical awareness and teaching the Colombian armed conflict: An analysis of social sciences textbooks (1984-2016), explains theoretical elements to understand the importance of connecting historical awareness to the epistemological, ethical and political aspects encountered while teaching recent history at the school level. In the discussion, the authors begin by analyzing the challenges involved in teaching violent pasts; furthermore, they define historical conscience, considering the elements that comprise it. Finally, the potential of establishing a relationship between historical consciousness and teaching recent history is discussed. The last section includes the main points of the work, emphasizing the importance of building theoretical bridges to understand various historical narratives within a school setting.

Keywords: education, history teaching, political education, school, social problem.
Este trabalho, produto da tese de doutoramento intitulada $\mathrm{La}$ conciencia histórica y la enseñanza del conflicto armado colombiano: un análisisis de los libros de texto escolar de ciencias sociales (1984-2016), procura expor elementos teóricos que permitem compreender a importância de ligar a categoria de consciência histórica a desafios epistemológicos, éticos e políticos que enfrentam o ensino da história recente na escola. A fim de desenvolver a discussão, primeiro são analisados os desafios envolvidos no ensino de passados violentos; em uma segunda frente, a definição de consciência histórica é exposta, tendo em conta os elementos que a compõem; em um terceiro ponto, o potencial de estabelecer uma relação entre a categoria de consciência histórica e o ensino da história recente é discutido. A última seção reúne os principais pontos do que foi exposto, enfatizando a importância de tecer pontes teóricas para compreender diversas narrativas históricas no contexto escolar.

Palavras-chave: educação, ensino de história, formação política, escola, problema social. 


\section{Introducción}

El contexto colombiano está marcado de manera significativa por una historia reciente trazada alrededor del conflicto armado que durante décadas ha perpetuado la violencia física y simbólica contra diversos sectores de la sociedad. Tramitar la comprensión de estos pasados cargados de profundo dolor se articula a las maneras como, desde diferentes agentes, instituciones o discursos, se han puesto a circular versiones del conflicto y la violencia. En ese camino, una de las principales responsabilidades en el reconocimiento de las anteriores problemáticas se ubica en la escuela, que, como lugar donde se realiza gran parte de la transmisión de lo que se debe recordar u olvidar (Raggio, 2017), asume la tarea de seleccionar cómo enseñar temas de gran impacto o trauma para la sociedad. Así, como lo pregunta De Amézola (2008), los contenidos cambian y la información sobre la historia en la escuela aumenta, pero ¿esto es suficiente?, y más aún, ¿es la única manera para reflexionar sobre estos hechos de gran impacto social?, ello teniendo en cuenta que la disciplina histórica no es un diccionario de conceptos o la memoria social no es solo un asunto del recuerdo individual.

Particularmente, los desafíos que atraviesa la escuela colombiana para la enseñanza de la historia reciente se instalan desde la lucha por la incorporación de diversas memorias que den cuenta de los procesos históricos que constituyen los pasados en conflicto. Para Rubio (2016), la tendencia que han tenido los currículos de naturalizar la violencia, eliminar la responsabilidad que tienen las clases políticas sobre su continuidad y desvincular a los sujetos en la transformación de la realidad, ha sido producto de la perpetuación de narrativas hegemónicas que reconocen de manera parcial las memorias sociales que atraviesan el conflicto. En ese sentido, aunque la necesidad para contextos como el colombiano de incorporar la enseñanza de la historia reciente sea un llamado actual y su importancia radique en los aportes que hace a un proyecto epistemológico, político y social de comprensión de los hechos traumáticos, esta:

[no deja de tensionar] la lógica de las tradicionales disciplinas escolares ocupadas del abordaje histórico y social, a la vez que conflictúa la escuela al incorporar unos contenidos problemáticos y complejos ligados a sucesos de reciente ocurrencia, que desafían los currículos prescritos y que [...] cuestionan los órdenes morales de la sociedad y de la comunidad escolar en particular. (Arias, 2015, pp. 33-34)

Lo anterior se ha expresado en la escuela colombiana desde la amplia brecha entre la complejidad del conflicto y los contenidos que los currículos y la normatividad asume deben ser enseñados, por mencionar incipientemente una de sus dificultades. Problemáticas fácilmente rastreables como la mención de temas inconexos sobre el conflicto o, la violencia en los lineamientos y estándares ${ }^{3}$ o la falta de articulación con las historias orales y de vida de diversos actores o víctimas del conflicto, reflejan el poco interés de algunos sectores por promover el tema en la escuela, la falta de compromiso de las políticas nacionales y el poco entendimiento de un contexto que, a pesar de su impacto, no es todavía una prioridad para la enseñanza.

Las mencionadas demandas se articulan a la necesidad de comprender la importancia del aprendizaje histórico y su aporte en contextos como el colombiano, el cual no se restringe a la transmisión escolar (Valls, 2008), sino que busca integrar el saber de la historia (científico o cotidiano) en la experiencia humana (Schmidt, 2017). Sus particularidades se vinculan a la lucha por la imposición de narrativas históricas que promueven formas del recuerdo, el olvido y la comprensión histórica. Así, la enseñanza del conflicto armado refleja las disputas por los usos del pasado, con orientaciones políticas, epistemológicas y estéticas determinadas, pero, a su vez, se enfrenta a las diversas subjetividades de quienes viven la historia en su cotidianidad y proyectan con ella futuros determinados.

Considerar la enseñanza del pasado refleja las disputas por el sentido, las cuales inciden en la instalación de proyectos políticos y sociales en el presente y futuro, determina un reto académico y político para pensar las categorías que posibiliten tanto su entendimiento como la visibilización de los caminos para potenciar su enseñanza en la escuela. Para ello, es preciso analizar el aprendizaje histórico como la capacidad de aportar a la constitución de una conciencia histórica, es decir, que más allá de la neutralidad aparente de sus contenidos, la enseñanza del pasado determina en los sujetos y las colectividades formas en que el pasado es comprendido para los proyectos del presente y el futuro de la sociedad.

Según el punto de partida expuesto, el presente artículo busca adelantar una discusión teórica de cómo los elementos de la categoría conciencia histórica pueden ser integrados para la comprensión de la enseñanza de

\footnotetext{
3 A pesar de la magnitud e intensidad del conflicto, el sistema educativo colombiano apenas vislumbra desde la normatividad educativa los primeros pasos para la enseñanza de los pasados violentos y traumáticos de la sociedad colombiana. Por ejemplo, los Lineamientos en Ciencias Sociales (MEN, 1998) solo mencionan en algunos ejes la promoción de derechos y construcción de paz; igualmente, los Estándares Básicos de Competencias en Ciencias Sociales (MEN, 2004) apenas tratan temas puntuales para los grados noveno, décimo y undécimo relacionados con hechos políticos de mediados del siglo XX (9 de abril, Frente Nacional), crisis del bipartidismo, movimientos guerrilleros en Colombia y desplazamiento forzado de poblaciones, lo que evidencia que las temáticas que existen solo se aproximan a la enunciación de contenidos. Si bien este no es el único panorama que puede abordar el problema en la escuela, sí presenta un aporte de gran incidencia en la consolidación de políticas educativas en el tema, ya que desde los lineamientos o estándares se constituyen currículos y planes institucionales, y para el caso que convoca, contenidos de libros de texto, determinando, con gran éxito, el privilegio de difundir ciertas miradas de los temas del pasado en conflicto en la escuela.
} 
la historia reciente. Esta discusión se adelanta desde la tesis doctoral titulada La conciencia histórica y la enseñanza del conflicto armado colombiano: un análisis de los libros de texto escolar de ciencias sociales (1984-2016) y ha tomado gran relevancia a partir de las pocas investigaciones que en Colombia se han realizado alrededor de conciencia histórica, a pesar de su potencia para develar cómo la enseñanza de los pasados promueve formas de interpretación y proyectos futuros a nivel político y social.

Para desarrollar la discusión, primero se analizarán los retos que asume la enseñanza de la historia reciente como escenario de lucha epistemológica y política por vincular diversas narrativas del pasado; en un segundo momento, se expondrá la definición de conciencia histórica, teniendo en cuenta los elementos que la componen; en un tercer momento, se discute el potencial que tiene vincular la categoría de conciencia histórica a la enseñanza de la historia reciente; finalmente, el último apartado recoge los principales puntos de lo expuesto y el potencial de las discusiones adelantadas para indagar sobre las narrativas históricas de los pasados violentos en el escenario escolar.

\section{Los retos de la enseñanza de la historia reciente}

La enseñanza de la historia reciente se ha constituido desde el interés de abordar los pasados violentos o conflictivos en los escenarios escolares. Su inserción ha puesto sobre la mesa el reto de cómo la disciplina escolar organiza su reconocimiento en el currículo, la consolidación de nuevas estrategias didácticas para su aprendizaje y el papel en la formación política de los actores escolares. Lo anterior se ha gestado desde un campo político y epistemológico más amplio que ha abordado la historia reciente, ello con la finalidad de pensar nuevas conceptualizaciones y formas de reivindicación política para superar diversos sucesos violentos y dolorosos en la sociedad.

La comprensión conceptual de la historia reciente y su inserción como preocupación en la enseñanza es posible ubicarla después segunda mitad del siglo XX, cuando se propició un desplazamiento epistemológico y político de una concepción del pasado moderno hacia el tiempo presente (Mudrovcic, 2005). Después de la Segunda Guerra Mundial y las discusiones por el fracaso del proyecto moderno, se permitió la incorporación de la historia reciente $^{4}$ como una categoría para abordar las diferentes experiencias del tiempo humano, integrar la memoria al

4 Aunque para la presente investigación se usará el término historia reciente, desde los aportes de investigadoras como Mudrovcic (2005), Rubio (2016) y Herrera y Pertuz (2016), otras variaciones del término como historia del tiempo presente, pasado reciente o historia del presente no son contradictorios con el concepto, ya que buscan abordar la integración de diversas temporalidades humanas (como por ejemplo entre memoria e historia). Estos términos, en consecuencia, reflejan "el carácter abierto a discusión de esta categoría temporal” (Rubio, 2016, p. 111). conocimiento histórico, visibilizar el papel del historiador como parte del pasado y buscar la apertura de espacios para pensar diversos hechos de gran impacto y trauma social ${ }^{5}$.

Con lo anterior, las diferentes narrativas del pasado ya no eran de uso exclusivo de los gremios profesionales, sino que se insertaron en el uso público de la historia, caracterizado por el interés de posicionar el saber histórico, asumiendo una dimensión deliberativa y comunicativa, la cual sirviera como insumo para estudiar la autoconciencia de los sujetos respecto a los sucesos violentos (Habermas, citado por Cuesta, 2011). Lo anterior ayudó a que la historia reciente también se definiera como el estudio de los pasados próximos cargados de una singular violencia y dolor, que son asumidos como traumáticos para los individuos y la sociedad (Kriger, 2011; Figueroa e Iñigo, 2010).

De ahí que el contexto escolar, como promotor de diversas narrativas históricas, se consolide en un escenario de lucha por la incorporación de los temas de la historia reciente al posicionar desde la enseñanza y aprendizaje la importancia de los temas del pasado en conflicto, el rigor científico que debe tener su abordaje y la necesidad de comprender las memorias que se entremezclan entre la identidad nacional, la rememoración de los pasados traumáticos y la reconstrucción de la historia oral (González y Pagés, 2014). Lo anterior se une con la importancia que ha suscitado la enseñanza del conflicto, con la tarea de lograr la articulación curricular de la investigación disciplinar, pedagógica y educativa (Sánchez y Rodríguez, 2008) y con las apuestas políticas que los diversos contextos requieren para abrir los escenarios de discusión y comprensión de los pasados violentos.

Las luchas necesarias para la incorporación de la historia reciente en la escuela se articulan a diferentes flancos que evidencian la complejidad que atañe su incorporación en el currículo. Un frente de importante desarrollo es el que asume la incorporación de la categoría de conciencia histórica para situar la enseñanza de la historia reciente como un proyecto epistemológico, político y pedagógico en torno a la comprensión del pasado, presente y futuro; así, enseñar las memorias, las violencias, el conflicto o los pasados traumáticos no solo implica la forma en que se presenta o no una temática en la escuela, sino, por

5 Con el fin de aportar a la discusión sobre la historia reciente, para Mudrovcic (2005), esta categoría se puede delimitar a partir de los fenómenos o acontecimientos que son recordados por al menos una de las generaciones que comparten el presente. Para la autora, una caracterización de la historia del presente debe tener en cuenta la delimitación de lapsos acotados, replantear el papel de objetos y sujetos y tener en cuenta que se solapan diferentes temporalidades de la experiencia. Esta definición aporta en la delimitación teórica y metodológica del tiempo presente ya que evidencia el papel de los sujetos en la historia, las múltiples capas que se cruzan para entender la temporalidad humana (experiencia vivida y tiempo universal, por ejemplo) y despoja al historiador de su separación del objeto. 
el contrario, se articula a un proyecto que busca indagar sobre el pasado enseñado y aprendido por los sujetos y las colectividades, las interpretaciones que la ciencia histórica tienen en el presente sobre los pasados contados en la escuela y las apuestas éticas, políticas y morales que permiten a los sujetos y sociedades tomar decisiones en el futuro a partir de su conocimiento de la historia.

Con el reto expuesto, a continuación se ampliará en la conceptualización de la categoría de conciencia histórica, llevando posteriormente a puntualizar sobre su potencial para entender la enseñanza de la historia reciente en la escuela.

\section{La conciencia histórica y sus elementos}

La conciencia histórica, como categoría enmarcada en los estudios sobre la enseñanza y el aprendizaje de la historia ${ }^{6}$, surge desde el interés de diversos contextos académicos, especialmente en Alemania, por analizar el papel de los sujetos y las sociedades en la circulación del conocimiento histórico (Cataño, 2011). Los trabajos del historiador Jörn Rüsen ${ }^{7}$ en los 80 permitieron acuñar el término de

6 Los desarrollos de la categoría de conciencia histórica son el producto de diversas fuerzas y corrientes teóricas que permitieron, posteriormente, su llegada al campo educativo. Autores como Gadamer (1993) y Aron (1983) fueron las primeras referencias sobre el tema, anunciando una conciencia del sujeto sobre su historia. Posteriormente, el marco de la historia social crítica, los trabajos de Jürgen Kocka, Hans-Ulrich Wehler, Kosselleck y Habermas entre otros (Millán, 2002) plantearon la importancia de la historiografía como campo que ofrece explicaciones frente al pasado, la vinculación de las demandas de la historia en los conflictos sociales y el uso de la historia en la esfera pública se entrelaza a las maneras como los sujetos y las colectividades aprenden y usan el conocimiento histórico. Esto se ha unido al interés desde la didáctica de la historia, la cual ha proporcionado a la discusión elementos de carácter teórico y metodológico para comprender la historia desde las prácticas de enseñanza aprendizaje en la escuela.

7 Jörn Rüsen es un historiador y filósofo alemán que se ha dedicado a indagar sobre la teoría de la historia y sus procesos de enseñanza. Entre sus mayores aportes se encuentran sus disertaciones alrededor de la racionalidad y objetividad de la ciencia histórica, las formas en que se desarrolla el pensamiento histórico, la constitución de las narrativas históricas y la consolidación de las dimensiones de la cultura histórica, entre otros. También se pueden destacar sus teorizaciones a partir del desarrollo de la matriz disciplinar que revela los elementos que constituyen el pensamiento histórico, las discusiones de la didáctica de la historia como lugar para analizar la enseñanza del pasado y el aporte del pensamiento histórico para superar el etnocentrismo y constituir identidades plurales. Su obra ha sido base para las investigaciones de algunos autores(as) como Tutiaux-Guillón (2003), González (2006), Lee (2006), Barca (2013) y Schmidt (2017) y proyectos de gran envergadura como Youth and History que en 1994 indagó los rasgos de conciencia histórica de jóvenes en veinticinco países europeos, al igual que su homólogo en Latinoamérica titulado Jovens diante da história que integró la conceptualización de la conciencia histórica y la cultura política para conocer cómo un grupo de jóvenes de Brasil, Uruguay, Argentina y Chile constituyen relaciones desde la enseñanza de la historia.

Cabe resaltar que en Colombia se conocen dos trabajos publicados desde la perspectiva del autor como es el de Cataño (2011) que desarrolla algunas categorías de la conciencia histórica analizando narrativas de la independencia en libros de texto escolar, y el de Pantoja, Loaiza y Posada (2013), que busca indagar cómo la formación de conciencia histórica en estudiantes de Licenciatura en Ciencias Sociales aporta a la enseñanza conciencia histórica para el análisis de las particularidades de la educación histórica. Los estudios se interesaron por analizar cómo se construyen y despliegan las diferentes temporalidades de lo humano (pasado, presente y futuro), su papel en la orientación y construcción de identidades, el desarrollo de valores morales, así como la vinculación de las concepciones temporales del sujeto a una cultura histórica determinada.

Para el autor, el problema de la enseñanza y el aprendizaje de la historia, y en especial el valor investigativo de la categoría de conciencia histórica, se establece desde una concepción que sitúa la didáctica de la historia en marcos que podrían superar la traducción o el transporte de los conocimientos históricos producidos científicamente (Rüsen, 2007a; 2009). En ese camino, la didáctica de la historia podría ser entendida por su estrecha relación con la ciencia histórica, ya que esta sería la encargada de analizar los asuntos relacionados con el aprendizaje del pasado en diferentes espacios como la escuela, los museos o los medios de comunicación (Rüsen, 2007a; 2009).

De tal forma, es posible entender la conciencia histórica como "la suma de las operaciones mentales con las que los hombres interpretan su experiencia de la evolución temporal de su mundo y de sí mismos, de forma tal que puedan orientar intencionalmente su vida práctica en el tiempo"8 (Rüsen, 2001, p. 57). La anterior definición pone en acento dos rasgos fundamentales en el desarrollo del concepto. El primero, en el carácter antropológico de la conciencia histórica, es decir, que todos los seres humanos al habitar un espacio en el mundo tienen una relación con el tiempo y actúan gracias a este; así, la conciencia histórica no es una meta que se alcance (Cerri, 2011) solamente con la adquisición de, por ejemplo, conceptos científicos, sino que es una condición propia de los sujetos que se expresa como un tipo de superávit de intencionalidades $^{9}$, ya que todos los seres humanos deben interpretar y actuar según las relaciones con el tiempo.

de la historia regional. En la actualidad, el campo de indagación de la conciencia histórica sigue suscitando gran interés en las ciencias de la educación; investigaciones como las de la profesora Graciela Rubio y el profesor Fabián González en Chile (conocido por la autora de este proyecto en su pasantía doctoral) utilizan estos referentes para analizar la narrativa histórica de escolares; igualmente, la propuesta de la investigadora Nelly Rodríguez Melo de la Universidad Nacional de Colombia busca integrar los principios de la conciencia histórica a los desarrollos sobre pensamiento histórico.

8 Traducción propia.

9 Para Rüsen (2001), los sujetos, por habitar en el mundo, tienen la necesidad de relacionarse con otros sujetos y con los objetos que los rodean. Estas relaciones son definidas como superávit de intencionalidades, las cuales se constituyen como la maneras fundamentales e indispensables con que cuenta el hombre para desarrollar sus relaciones en el mundo. En consecuencia, uno de los superávit de intencionalidades destacados por el autor es la relación de los seres humanos con el tiempo, el cual se consolida en una de las experiencias fundamentales por las cuales pasan todos los sujetos, ya que perciben y viven el tiempo de manera inevitable. Lo anterior permite afirmar que la conciencia histó- 
Como segundo elemento, se hace relevante señalar que la conciencia histórica es una operación que se instala en la vida práctica, ya que los sujetos proceden según viven el tiempo, enfrentan los cambios y principalmente se interpretan a sí mismos y al mundo para actuar según ciertas intenciones. De esta manera, las experiencias en el tiempo son inherentes a los sujetos, constituyéndose en inevitables; igualmente, la orientació $n^{10}$ permite actuar según un horizonte de en la vida práctica. Experiencia y orientación no están desarticuladas en la vida cotidiana, sino que su interacción es decisiva a la hora de interpretar y actuar según el tiempo histórico.

Evidenciar cómo es asimilada la experiencia y la orientación del tiempo histórico es posible gracias a que la conciencia historia se da en medio de una "concepción del cambio temporal que abarca el pasado, presente y futuro" (Rüsen, 1992, p. 29). Particularmente, los procesos mentales de la conciencia histórica permiten que los sujetos conozcan la alteridad del pasado (no todo es como el presente), generen marcos de interpretación para entender el presente y abran el futuro a las posibilidades de cambio ${ }^{11}$. Desde la perspectiva de Rüsen (2007b; 2009), los detalles en los cuales se dan los procesos mentales de la conciencia histórica pueden ser explicados desde cuatro procedimientos básicos que, aunque son artificiales, dan cuenta de las relaciones de los sujetos con el aprendizaje de lo histórico:

- Se establece con el tiempo histórico una percepción que permite diferenciar el pasado del tiempo actual. Así, existen narrativas que por medio de los sentidos posibilitan asimilar la alteridad del pasado.

- Se producen operaciones en los individuos que les permite la interpretación del tiempo pasado, entendida

rica no es privativa de una cultura o de un momento de la historia, sino es una categoría antropológica, ya que de una u otra manera todos los sujetos y el mundo que habitan están insertos en diversas relaciones con el pasado (Cerri, 2011; Paul, 2016).

10 La orientación de las acciones humanas producto de la historia es una condición dada antes de la apropiación del sujeto. Toda relación con la historia está constituida por relaciones objetivas que determinan el rumbo que los sujetos siguen según la historia y determina la formación subjetiva (Rüsen, 2007a). Aunque existen condiciones de orientación ya dadas (punto que se aclarará más adelante con la definición de cultura histórica), el sujeto también desarrolla su creatividad para apropiar y utilizar dichas orientaciones en la vida práctica.

11 Para Rüsen (2007b), la complejidad que acarrea el abordaje de las diferentes temporalidades en la conciencia histórica le permite diferenciar su interpretación del concepto de memoria histórica, ya que este último solo hace énfasis en la operación de traer el pasado al presente (pasado biográfico o histórico), sin abordar con profundidad las expectativas que se pueden tener hacia el futuro. Es por tanto que la conciencia histórica se consolida como marco interpretativo más sofisticado (Cataño, 2011) por abarcar las diferentes relaciones del tiempo en los sujetos. Esto no quiere decir que la memoria no sea una operación fundamental para la conciencia histórica; sin esta el pasado no tendría una significación en el presente desde la rememoración, por lo cual el sujeto no se situaría desde las relaciones históricas. como movimiento de la humanidad. Se interpreta el pasado desde ciertas categorías, discursos o visiones de mundo.

- Las relaciones con el pasado permiten una orientación de la vida humana desde las interpretaciones que se generan, es decir, controla la intención de actuar en la vida práctica.

- La motivación está relacionada con las acciones a futuro. Su tarea es poner en práctica las orientaciones dadas en forma de intención. Marca el actuar del sujeto desde la voluntad, los impulsos, los intereses y las necesidades.

No obstante, la conciencia histórica "no emerge de la nada ni es fruto exclusivo de sus experiencias vitales autónomas" (Sánchez-Costa, 2013, p. 201), sino, por el contrario, se encuentra inserta en marcos culturales y sociales en los cuales se ha determinado una forma de concebir y vivir las relaciones con el pasado definida como cultura histórica ${ }^{12}$. El mencionado concepto permite entender cómo las relaciones subjetivas que constituye la conciencia histórica están insertas en relaciones más amplias que incluyen agentes e instituciones en los cuales se establecen referentes comunes y se le da sentido al pasado en una cultura determinada.

La cultura histórica se entiende como "el modo concreto y peculiar que tiene una sociedad de entender la temporalidad y de relacionarse con su pasado" (Sánchez-Costa, 2013, p. 203). Las formas en que se encarna parten desde diversos recursos, prácticas sociales y discursos en los cuales los sujetos y las sociedades generan interpretaciones, consolidan procesos de transmisión, objetivan las relaciones con lo histórico y transforman el pasado (2013). Lo anterior es posible gracias a que la cultura histórica es "la articulación práctica y operativa de la conciencia histórica en la vida de la sociedad" (2013), que significa, por un lado, la capacidad de los sujetos y su conciencia para crear $\mathrm{y}$ trascender hacia estructuras objetivas que materializan las relaciones con el tiempo; por otro, la capacidad de configurar subjetividades, ya que la conciencia histórica no se forma fuera de una atmósfera cultural determinada ${ }^{13}$. En suma, la cultura histórica y la conciencia histórica son dos caras de la misma moneda (Schöneman, citado por

\footnotetext{
12 La categoría de cultura histórica ha suscitado un amplio campo de estudios, el cual, si bien no siempre ha estado atado a las conceptualizaciones de conciencia histórica, sí ha buscado evidenciar las relaciones de los sujetos y las colectividades con los diferentes regímenes históricos. Esta categoría surgió del interés social o boom por indagar el uso público la historia y la memoria social, así como por la necesidad de realizar apuestas teóricas que permitan entender las relaciones del pasado desde su carácter empírico y objetivo.

13 Lo mencionado tampoco indica que todos los seres humanos cuenten con una conciencia histórica igual o predeterminada en todas sus relaciones por un horizonte cultural, por el contrario, el sujeto cumple un papel importante en los procesos de creación y reflexión de las experiencias temporales.
} 
Sánchez-Costa, 2013), donde la presencia de una determina la configuración de la otra y viceversa.

La configuración de la cultura histórica se da en el marco de la memoria histórica, la cual permite traer referentes del pasado al presente para dar forma a la conciencia más allá de las biografías de los sujetos. Particularmente, la memoria histórica integra al sujeto elaboraciones del pasado que contienen formas normativas, concepciones humanas, tradiciones e identidades modeladas en el marco de la cultura histórica. Es así como los entramados de la cultura histórica aportan los marcos externos que orientan las formas sociales de relacionarse con el tiempo y consolidan aspectos internos como la identidad ${ }^{14}$ de los sujetos. Para Rüsen (1994, 2001 y 2013), este proceso se hace desde las diferentes dimensiones como relaciones empíricas y objetivas que forman elaboración y circulación de la historia. Entre los rasgos que se pueden identificar se encuentran:

- Dimensión cognitiva. Hace referencia a todo saber o conocimiento sobre el pasado humano que se constituye en pilar de la interpretación del tiempo histórico. Los criterios de sentido que se delimitan en el espacio cognitivo se hacen a partir de la verdad, lo cual tiene relación con la capacidad de justificar las narrativas como legítimas ${ }^{15}$.

- Dimensión política. Hace hincapié en las luchas por el poder político. Aquí se expresan todas las disputas relacionadas con la dominación y legitimación de las relaciones de poder que utilizan las estructuras de lo histórico.

- Dimensión estética. Concreta las narrativas del pasado desde relatos textuales expresados en diversas formas de representación. Uno de sus criterios de formación es la coherencia de su narrativa, la cual está dada por los marcos normativos que le dan sentido a lo histórico; en esta dimensión, los sujetos se acercan de manera sensorial a la comprensión del pasado.

14 La identidad, como la interpretación que hace el sujeto de sí mismo y de su vida (Sánchez-Costa, 2013) en relación con un grupo de pertenencia, no se da únicamente como acto de reflexión individual, sino que está inserto en una narrativa histórica que construye un nosotros común que circula en la memoria histórica y es asumido por los sujetos. La identidad de las colectividades es un rasgo de gran influencia en el modelamiento de la conciencia histórica, ya que esta delimita experiencias y orientaciones tanto en las operaciones mentales como desde la cultura histórica para dar unidad y continuidad al grupo (Cerri, 2011). Uno de los mayores ejemplos de lo anterior es la constitución de la identidad nacional, donde el Estado, desde esferas como la educación, modela las estructuras mentales, sociales y culturales que responden al sentimiento de unidad y hegemonía nacional, generalizando formas de dominación de ciertos rasgos de la conciencia histórica. Así, los diferentes elementos que componen la conciencia histórica se tornan en armas en la pugna por definir el rumbo de las colectividades (Arias, 2017; Cerri, 2011).

15 La pretensión cognitiva de verdad es uno de los sentidos más importantes de la cultura histórica, ya que sin esta no se podría actuar desde referentes del pasado.
Las anteriores dimensiones de la cultura histórica son básicas para develar y caracterizar la memoria que interviene en la formación de conciencia histórica. Ninguna de las dimensiones puede subsistir sin tener relación con la otra (de dominio, choque o complemento). Igualmente, dependiendo el punto de análisis, se pueden establecer otras dimensiones que permiten develar la influencia de diversas memorias en la constitución de la cultura histórica; casos particulares serían los del análisis de la ciencia histórica que incluye la dimensión moral como la responsabilidad de la labor científica y la religiosa desde la trascendencia humana. También el análisis del pensamiento histórico que incluye la dimensión semántica o material y busca marcar las diferencias entre pasado e historia y la retórica o moral que da una lección u orientación en la vida práctica (Rüsen, 2009; 2013) ${ }^{16}$.

Las anteriores dimensiones no se dan alejadas del sujeto. Por el contrario, su impacto en la conciencia histórica posibilita diversas relaciones con el pasado en relación con los modos en los que los sujetos hacen conexión con los sucesos históricos (Day, citado por Paul, 2016). Evidenciar lo mencionado parte, por ejemplo, de comprender que la percepción revela narrativas frente la alteridad del pasado, las cuales trascienden la memoria biográfica (memorias históricas provenientes de la dimensión semántica o material); la interpretación convierte al pasado en histórico desde marcos de análisis disponibles en el presente (la historiografía) y la orientación y motivación representan el futuro como expectativa de vida personal, movilizando objetivos e intenciones (poder, legitimidad del Estado, responsabilidad de la acción histórica) insertas en el espacio cultural (Rüsen, 2007b).

Para captar las anteriores manifestaciones de la conciencia histórica, se utiliza la narrativa histórica, la cual sintetiza la instancia comunicativa por la cual los relatos del pasado son socializados y apropiados por el sujeto. La narrativa histórica se diferencia de otras formas narrativas al cumplir la función de organizar las tres dimensiones de la temporalidad humana (Rüsen, 2001; Cerri, 2011). Al narrar el pasado, el presente y el futuro se constituyen como una unidad continua y coherente según el sentido de la historia personal y social ${ }^{17}$, lo que proporciona coordenadas para interpretar la historia y orientar elementos o momentos de la vida práctica (Cerri, 2011).

La narrativa histórica, como producto tangible de la unidad y orientación de las temporalidades humanas, en palabras de Herman Paul (2016), se configura desde una relación estética con el pasado. Para el autor, esta no se

\footnotetext{
16 Paul (2016), desde la perspectiva de Rüsen, aporta elementos adicionales para comprender las dimensiones de la conciencia histórica relacionadas con lo material, epistemológico, moral y político y estético. 17 Esto no oculta que, aunque la narrativa histórica privilegie una estructura de continua, lineal y única para dar sentido a la realidad, esta se encuentra cruzada por diferentes tiempos históricos que dan cuenta de sus cambios, transiciones y rupturas (Mudrovcic, 2005)
} 
da en el sentido de belleza de lo histórico, "sino porque utilizan formas de relato o, en términos más técnicos, patrones narrativos, que modelan la forma en que las personas miran el pasado" (Paul, 2016, p. 107). Lo dicho indica que la relación estética es una relación sensorial (Rüsen, 1994), ya que los sujetos se acercan o producen diversas expresiones narrativas en las cuales los relatos del pasado se manifiestan. Estas no solo son formas discursivas, sino que pueden estar condensadas en imágenes, palabras o símbolos como abreviaciones narrativas (Cerri, 2011).

Igualmente, las expresiones de la narrativa histórica desde su componente estético buscan la incorporación de ciertos patrones normativos de la conciencia histórica (Cerri, 2011). Lo anterior se da porque los relatos que conforman la narrativa histórica están atados, como ya se analizó, a una cultura determinada. La influencia de estos patrones permite modelar la vida en el mundo al orientar o justificar desde la historia las acciones de los sujetos, establecer juicios morales e interpretar el mundo de forma retrospectiva (Paul, 2016; Cerri, 2011). En ese sentido, es preciso afirmar que:

La forma de relato ejerce poder: un poder discursivo que determina, al menos en parte, la forma en que las personas interpretan sus vidas. Cuando más personas modelan sus vidas de acuerdo con el patrón del hombre hecho a sí mismo o de la heroína romántica, más poder ejercerá ese patrón sobre ellas. (Paul, 2016, p. 109)

Así, la conciencia histórica por intermedio de sus narrativas expresa las intenciones, expectativas, intereses de agentes o agencias productoras o recontextualizadoras de discursos sobre el pasado que buscan mantener e imponer ciertas perspectivas para su comprensión y uso. En ese sentido, la narrativa histórica se hace evidente en escenarios como las escuelas (Rüsen 1994; Sánchez-Costa, 2013), lo que determinaría las "funciones de la enseñanza, del entretenimiento, de la legitimación, de la crítica, de la distracción, de la ilustración y de otras maneras de memorar" (Rüsen 1994) desde la integración de ciertos tipos u órdenes en los cuales está constituida la conciencia histórica.

Los elementos de la conciencia histórica estarían presentes en la forma en la que se enseña, aprende o transforma las narrativas del pasado que circulan en la escuela, potencial que, como se verá a continuación, permite develar o integrar apuestas políticas, epistemológicas y éticas que constituyen la enseñanza de la historia reciente en la escuela.

\section{La enseñanza de la historia reciente y su vinculación con la conciencia histórica}

La enseñanza de la historia reciente establece una relación estrecha con el desarrollo de la conciencia histórica, ello al brindar herramientas para la comprensión de las diferentes pugnas que los pasados en conflicto abordan (tiempos cronológicos, memoriales o biográficos) y aportar para la orientación de la vida práctica en busca de proyectos sociales o políticos que visibilicen diferentes apuestas al futuro. Para que exista una formación histórica en temas como los de la historia reciente es necesario que la escuela promueva la comprensión del sentido del cambio temporal (Rüsen, citado por Schmidt, 2017), lo anterior presupone hacer conexiones entre la historia personal y las dimensiones de la cultura histórica para constituir narrativas más complejas que reconozcan la alteridad del pasado (historia biográfica, hecho histórico), proporcionen herramientas para comprender el presente (ciencia histórica como una explicación de la realidad) y formulen estrategias orientativas y motivadoras para actuar (propuestas críticas y de transformación).

Así, la conciencia histórica amplía sus horizontes cuando la enseñanza de la historia desarrolla la capacidad de pensar históricamente ${ }^{18}$, lo cual hace referencia a la integración de saberes (por ejemplo, los de la ciencia histórica) en la orientación de la vida práctica (Cerri, 2011) ${ }^{19}$. Aquí es indispensable que la comprensión del sentido histórico esté acompañada de la posibilidad no solo de ampliar el aprendizaje de contenidos, sino que los sujetos se acerquen a diversas relaciones con el tiempo para integrar saberes escolares y personales a un entendimiento múltiple del pasado.

En consecuencia, la narrativa histórica sería fundamental en el desarrollo del pensar histórico de los pasados recientes, ya que este es el vehículo por el cual maestros, estudiantes y material escolar comunican sus diversas relaciones con el pasado. Sin embargo, no basta solo con narrar el pasado conflictivo en el ámbito escolar, es preciso que se le sumen procesos de enseñanza y aprendizaje

\footnotetext{
18 Desde múltiples herramientas teóricas se ha visibilizado en los contextos educativos y pedagógicos el análisis del pensar históricamente, y teniendo en cuenta los procesos de enseñanza-aprendizaje de la historia y el uso de este saber para orientar la vida práctica de los sujetos, donde temas como el pensamiento histórico (Prats, 2000; Campos, Chacón y Rodríguez, 2007; Santisteban, González y Pagés, 2010;), la didáctica crítica (Cuesta, 2011 y 2014), la conciencia histórica (Rüsen, 1992, 1994 2001, 2007, 2013), el tiempo histórico (Pagés, 1998; Pagés y Santisteban, 2010) pedagogía de la memoria (Rubio, 2012 y 2016; Herrera y Pertuz 2016) y la enseñanza de la historia reciente (Arias, 2017) se consolidan en apuestas para la comprensión de una enseñanza de la historia atenta a los nuevos regímenes de historicidad, los pasados en conflicto, las diferentes temporalidades de lo humano, las diversas memorias colectivas o subjetivas y las apuestas éticas y políticas que disputan el sentido de la realidad.

19 Para Cerri (2011), la ampliación de la frontera de la conciencia histórica desde el pensar histórico debe desembocar en procesos que permitan la concientización histórica en el sentido freiriano, esto quiere decir que, a partir del cambio de las estructuras mentales de los sujetos, no solo se logre el desarrollo de formas de relación histórica que orienten la vida práctica, sino que lleguen a reconocer las diferentes identidades, consolidar un proyecto de intereses comunes y reconocer la producción de conocimiento histórico desde diversos campos.
} 
de la historia que partan de formatos y discursos claros, los cuales permitan la adquisición de competencias narrativas (Rüsen, 1992). Cuando a una narrativa histórica se le une la adquisición de competencias, significa que las prácticas de enseñanza de la historia deben permitir el desarrollo de "(a) habilidades para sentir la experiencia del pasado; (b) habilidades para interpretar la experiencia del pasado, la comprensión del presente y las expectativas del futuro; $y$ (c) habilidades para orientar y guiar el camino hacia el futuro" (Rüsen, citado por Sant et al., 2014).

Según lo anterior, la narrativa histórica de los pasados recientes debe contribuir al desarrollo de las competencias de percepción, interpretación y orientación que determinarían cómo se desencadenan los procesos de aprendizaje histórico en la conciencia histórica. Igualmente, son portadores de narrativas que elaboran modalidades de relación con el pasado, producto de una cultura histórica, observando vínculos de orden material, epistémica, política y moral, los cuales permitirían determinar un sentido particular de la historia y, principalmente, de cómo en una cultura escolar es entendido y usado el pasado. Aunque en la vida práctica estas relaciones no se diferencian, a nivel teórico su particularización permite analizar las fuerzas que confluyen en la configuración de una idea del pasado en el ámbito escolar.

La conjugación de los anteriores elementos también permitiría que los sujetos se acerquen y actúen bajo tipos de conciencia histórica, los cuales determinan ciertas relaciones desde la memoria histórica, la identidad y las perspectivas de futuro. La educación y la narrativa histórica que es enseñada serían los vehículos por los cuales se ponen a circular los tipos de conciencia histórica, expresando formas particulares en que se integran los referentes del pasado a la totalidad de la vida práctica.

Para Rüsen (1992; 2007a), los tipos de conciencia podrían caracterizarse desde cuatro formas que son: tradicional, ejemplar, crítico y genético, las cuales permiten categorizar y analizar los diversos sentidos que toma la historia para los sujetos y la cultura. Teóricamente, cada tipo de conciencia se expresa en forma de narrativa y su constitución se da dependiendo de las maneras en que son tratadas y utilizadas las experiencias y los conocimientos del pasado en la orientación de la vida práctica (Rüsen, 1992). Las tipologías dan cuenta de diversas modalidades y complejidades en las cuales se elabora y comprende el pasado, estas pasan desde una idea de continuidad temporal (tradicional) a una concepción más dinámicas del tiempo (genética); no obstante, en su análisis práctico las tipologías aparecen mezcladas de forma compleja (no se encuentran de manera pura), además de que ninguna puede existir sin algún elemento de la otra, siendo necesario establecer qué tipo de conciencia podría predominar y cuáles son las relaciones que se establecen allí (Rüsen, 1992 y 2007a).
Según lo anterior, cada tipología sería entendida así:

- Tipología tradicional. Los cambios del pasado en el sujeto y el mundo son vistos como entidades continuas, estables y duraderas. Su principal arraigo en el pasado está en la idea de tradición, la cual se presenta como una forma concreta y sucesiva de hechos que se arraigan a valores válidos y obligatorios. Asimismo, desde esta perspectiva la historia es vista como obvia, incuestionable o indecible, sustentando así identidades personales o colectivas desde un nosotros común que se arraiga en valores y en ideas de un destino ineludible para los sujetos.

- Tipología ejemplar. Ya no se trata de un pasado único para actuar en el presente como en el caso tradicional, sino que este se estructura desde diversas relaciones que son asimiladas como reglas, puntos de vista o principios. Así, por medio de ejemplos históricos, se transmiten formas de entender, actuar y visibilizar los cambios del pasado que serán reglas aplicadas a la vida práctica.

- Tipología crítica. Para una constitución de identidades y sentidos de la historia en el modo crítico, se rompe con los conceptos y categorías establecidas, procurando generar distanciamiento y resistencia con las ideas del pasado que se han establecido como únicas.

- Tipología genética. El pasado también es contemplado desde la oportunidad del cambio que permita superar y mejorar las condiciones de vida existentes. Aquí la historia es entendida como una instancia dinámica que permite proyectar cambios para el presente y el futuro.

\section{A modo de cierre}

La enseñanza de la historia reciente se define como un campo de tensión que oscila entre las visiones hegemónicas de la memoria que asumen el conflicto como algo inevitable y natural, y los desarrollos teóricos recientes que buscan integrar la historia y memoria para comprender diversas narrativas del conflicto y establecer apuestas para superarlo. No obstante, es desde las nuevas perspectivas para comprender la enseñanza de los pasados en conflicto que se instala como común denominador la importancia de la integración de diversas narrativas, las cuales se conectan tanto con las formas en que la conciencia histórica construye las relaciones entre el pasado, presente y futuro como con el análisis de la cultura histórica que determina los conocimientos legítimos sobre el tema en el ambiente escolar.

En ese sentido, cobra relevancia la categoría de conciencia histórica para el estudio de los pasados recientes que son enseñados en la escuela, ya que permite analizar cómo los "saberes históricos son colocados en relación, 
ampliados, escogidos, modificados"20 (Cerri, 2011, p. 69), dando pistas sobre cómo se gestionan en la escuela las formas de rememorar tanto subjetivas como en una cultura determinando y sus implicaciones para tiempos futuros.

La inserción de la conciencia histórica para el análisis de la enseñanza de la historia reciente es un campo de naciente apertura en Colombia y cobra gran valor al dar elementos teóricos para discutir la importancia del aprendizaje histórico desde las formas de elaboración intersubjetiva como son la percepción, la interpretación y la orientación, así como su vínculo con diversas maneras de elaboración del pasado, entendidas desde las relaciones materiales, epistemológicas, políticas y morales que se disputan los significados y sentidos de la realidad histórica en la constitución, por ejemplo, de los tipos de conciencia histórica. Finalmente, aportan también elementos metodológicos sobre las posibles categorías de análisis investigativo del campo de la enseñanza de la historia reciente al tener en cuenta "la relación crítica entre memoria e historia dando paso a reflexiones que vinculan las posiciones teóricas epistemológicas con el poder político" (Rubio, 2012, p. 376).

\section{Referencias}

Arias, D. (2015). La enseñanza de la historia reciente y la formación moral. Dilemas de un vínculo imprescindible. Folios, 42, 29-41. https://doi.or$\mathrm{g} / 10.17227 / 01234870.42$ folios29.41

Arias, D. (2017). Relatos de nación y escuela. Colombia en los imaginarios de docentes en formación. Bogotá: Universidad Distrital Francisco José de Caldas. https:// doi.org/10.14483/9789585434165

Aron, R. (1983). Dimensiones de la conciencia histórica. Ciudad de México: Fondo de Cultura Económica.

Barca, I. (2013). Conciencia histórica: pasado y presente en la perspectiva de los jóvenes en Portugal. Clío y Asociados. La historia enseñada, 17, 16-26. https://doi. org/10.14409/cya.v1i17.4170

Campos, D., Chacón, A. y Rodríguez, N. (2007). Orientaciones curriculares para el campo de pensamiento histórico. (Colegios públicos de excelencia para Bogotá). Serie Cuadernos de Currículo. Bogotá: Alcaldía Mayor de Bogotá; Secretaría de Educación Distrital.

Cataño, C. (2011). Jörn Rüsen y la conciencia histórica. Historia y Sociedad, 21, 221-243.

Cerri, L. (2011). Ensino de história e consciencia histórica. Implicações didáticas de uma discussão contemporânea. Rio de Janeiro: Editora FGV.

Cuesta, R. (2011). Historia con memoria y didáctica crítica. Con-ciencia Social: Anuario de Didáctica de la Geografia, la Historia y las Ciencias Sociales, 15, 15-30.

Cuesta, R. (2014). Genealogía y cambio conceptual. Educación, historia y memoria. Archivos Analiticos de Política Educativa, 22.
De Amézola, G. (2008). Currículo oficial y memoria. El pasado reciente en la escuela argentina. Enseñanza de las Ciencias Sociales, 7, 47-55.

Figueroa, C. e Iñigo, N. (2010). Reflexiones para una definición de historia reciente. En M. López, C. Figueroa y B. Rajland (eds.), Temas y procesos de la historia reciente de América Latina (pp. 13-34). Santiago de Chile: Clacso.

Gadamer, H. (1993). El problema de la conciencia histórica. Madrid: Editorial Tecnos.

González, M. y Pagés, J. (2014). Historia, memoria y enseñanza de la historia: conceptos, debates y perspectivas europeas y latinoamericanas. Historia y Memoria, 9, 275-311. https://doi. org/10.19053/20275137.2941

Herrera, M. y Pertuz, C. (2016). Educación y políticas de la memoria sobre la historia reciente en América Latina. Revista Colombiana de Educación, 71, 79-108. https://doi.org/10.17227/01203916.71rce79.108

Kriger, M. (2011). La enseñanza de la historia reciente como herramienta clave de la educación política. Persona y Sociedad, 3, 29-52.

Lee, P. (2006). Em direção a um conceito de literacia histórica. Educar em Revista, 22, 131-150. https://doi. org/10.1590/0104-4060.403

Ministerio de Educación Nacional (1998). Lineamientos Curriculares de Ciencias Sociales. Bogotá: Ministerio de Educación Nacional.

Ministerio de Educación Nacional (2004). Estándares Básicos de competencias en ciencias sociales. ¡Formar en ciencias el desafío! Bogotá: Ministerio de Educación.

Millán, J. (2002). El contexto de la historia social crítica en la Alemania contemporánea. En J. Kocka, Historia social y conciencia histórica (pp. 11-40). Madrid: Marcial Pons.

Mudrovcic, M. (2005). Historia, narración y memoria: los debates actuales en filosofía de la historia. Madrid: Akal Ediciones.

Pagés, J. y Santisteban, A. (2010). La enseñanza aprendizaje del tiempo histórico en la educación primaria. Cadernos Cedes, 30(82), 278-309. https://doi. org/10.1590/s0101-32622010000300002

Pantoja, P., Loaiza, Y. y Posada, R. (2013). Ecos del ayer, el hoy y el mañana: la conciencia histórica como propuesta en la formación de licenciados en ciencias sociales. Revista Latinoamericana de Estudios Educativos, 1(10), 136-157.

Paul, H. (2016). La llamada del pasado. Claves de la teoría de la historia. Zaragoza: Instituto Fernando El Católico. https://doi.org/10.26754/ojs_historiografias/ hrht.2017132355

Prats, J. (2000). Dificultades para la enseñanza de la historia en la educación secundaria: reflexiones ante la situación española. Revista de Teoría y Didáctica de las Ciencias Sociales, 5, 71-98.

Raggio, S. (2017). ¿Historia o memoria en las aulas?

20 Traducción propia 
Rubio, G. (2012). El pasado reciente en la experiencia chilena. Bases para una pedagogía de la memoria. Estudios Pedagógicos, 38(2), 375-396. https://doi. org/10.4067/s0718-07052012000200023

Rubio, G. (2016) Memoria hegemónica y memoria social. Tensiones y desafíos pedagógicos en torno al pasado reciente en Chile. Revista Colombiana de Educación, 71, 79-108. https://doi.org/10.17227/01203916.71rce109.135

Rüsen, J. (1992). El desarrollo de la competencia narrativa en el aprendizaje histórico. Una hipótesis ontogénica relativa a la conciencia moral. Propuesta Educativa, 7, 27-36.

Rüsen, J. (1994). ¿Qué es la cultura histórica? Reflexiones sobre una nueva manera de abordar la historia. En K. Füssmann, H. Grütter, H. y J. Rüsen (eds.), Historische Faszination. Geschichtskultur Heute (pp.3-26). Colonia, Alemania: Böhlau Verlag.

Rüsen, J. (2001). Razão histórica. Teoria da história: fundamentos da ciência histórica. Brasilia: Universidade de Brasilia.

Rüsen, J. (2007a). História viva. Teoria da história III: formas y funciones de conhecimento histórico. Brasilia: Universidade de Brasilia.

Rüsen, J. (2007b). How to make sense of the past: salient issues of metahistory. The Journal of Transdisciplinary Research in Souther Africa, 6(2), 169-221. https://doi. org/10.4102/td.v3i1.316

Rüsen, J. (2009). Como dar sentido ao passado: questões relevantes de meta-história. História da Historiografia, 2, 163-209.

Rüsen, J. (2013). La ciencia histórica como cultura histórica. En J. Lluis y F. Sánchez-Costa, $A$ vueltas con el pasado. Historia, memoria y vida (pp. 81-110). Barcelona: Universidad de Barcelona.
Sánchez-Costa, F. (2013). La fragua de la identidad: memoria, conciencia histórica y cultura histórica. En J. Lluis y F. Sánchez-Costa, A vueltas con el pasado. Historia, memoria y vida (pp. 185-211). Barcelona: Universidad de Barcelona.

Sánchez, M. y Rodríguez, S. (2008). Narrativa, memoria y enseñanza del conflicto armado colombiano: propuesta para superar las políticas de olvido e impunidad. En A. Serna y D. Gómez (Comps.), El papel de la memoria en los laberintos de la verdad, la justicia y la reparación (pp. 203-230). Bogotá: Universidad Distrital Francisco José de Caldas.

Sant, E., Pagés, J., Santisteban, A., González, N. y Oller, M. (2014). Narrativas y discursos: ¿cómo podemos analizar la competencia narrativa del alumnado en el aprendizaje de la historia? Revista Clío y Asociados. La historia enseñada, 18-19, 166-182. https://doi. org/10.14409/cya.v0i18/19.4742

Santisteban, A., González, N. y Pagés, J. (2010). Una investigación sobre la formación del pensamiento histórico. En: R. Ávila, M. Rivero y P. Domínguez (coord.), Metodología de la investigación en didáctica de las ciencias sociales (pp. 115-128). Zaragoza: Institución Fernando El Católico.

Schmidt, M. (2017). ¿Qué hacen los historiadores cuando enseñan la historia? Contribución de la teoría de Jörn Rüsen para el aprendizaje y el método de enseñanza de la historia. Clio y Asociados. La historia enseñada, 24, 26-37. https://doi.org/10.14409/cya.v0i24.6843

Tutiaux-Guillon, N. (2003). Los fundamentos de una investigación sobre la concepción de las finalidades cívicas y culturales del profesorado de geografía e historia. Objetivo de esta etapa. Enseñanza de las Ciencias Sociales, 2, 27-35.

Valls, R. (2008). La enseñanza de la historia y textos escolares. Bueno Aires: Libros del Zorzal. 